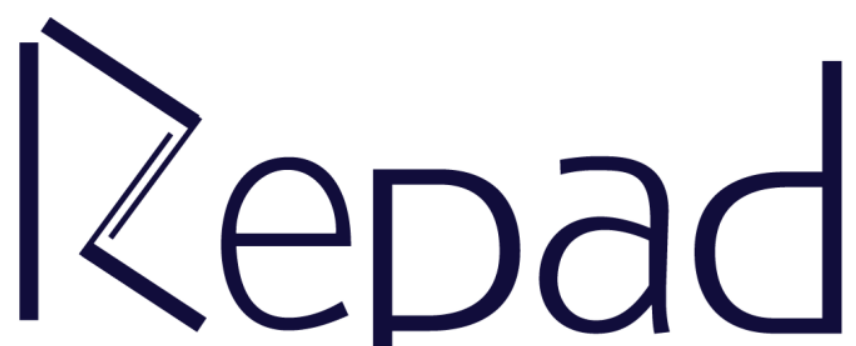

v. 5, n. 1, Janeiro-Abril/2021

Revista Estudos e

Pesquisas em Administração

(c) (i) This work is licensed under a Creative Commons Attribution 4.0 International License 


\title{
Análise da aplicação da ferramenta BSC na gestão pública em municípios de Mato Grosso sob a perspectiva dos gestores do planejamento
}

\author{
Beatriz Navarro Ferreira \\ beatriz.navarrof@gmail.com \\ ORCID 0000-0001-8929-6802 \\ Universidade Federal de Mato Grosso \\ Cuiabá, Mato Grosso, Brasil \\ Vergilio Prado Sogabe \\ ORCID 0000-0002-0799-8219 \\ Universidade Federal de Mato Grosso \\ Cuiabá, Mato Grosso, Brasil
}

\section{RESUMO}

O Balanced Scorecard (BSC) é uma das ferramentas mais utilizadas para medir o desempenho das organizações servindo de base para um sistema de medição e gestão estratégica, que tem sido implementada no setor público. No estado de Mato Grosso esse modelo passou a ser utilizado em 23 municípios a partir de uma iniciativa do Tribunal de Contas do Estado - TCE, por meio do Programa de Desenvolvimento Institucional Integrado - PDI. O objetivo geral do estudo é descrever como a ferramenta BSC tem contribuído para o aprimoramento da gestão pública municipal. Mais especificamente: a) identificar os fatores importantes na correta implementação da ferramenta BSC na literatura; b) identificar como a ferramenta está sendo aplicada nos municípios de MT; e c) descrever como a ferramenta é operacionalizada na percepção dos gestores do controle do planejamento municipal. A metodologia caracteriza-se como qualitativa básica, do tipo indutivo com caráter descritivo, por meio da aplicação de um questionário estruturado. Verificou-se como resultado principal o alinhamento do modelo de planejamento estratégico dos municípios com o modelo do BSC em suas respectivas dimensões. Por fim, constatou-se que na percepção dos respondentes a ferramenta foi implementada com êxito pelos municípios, favorecendo o aprimoramento da gestão pública municipal.

Palavras-chave: Balanced Scorecard, Alinhamento, Gestão Pública.

\section{Analysis of the use of BSC tool in public management in municipalities of Mato Grosso on the perspective of planning managers}

\begin{abstract}
The Balanced Scorecard (BSC) is one of the most used tools to measure the performance of organizations serving as the basis for a system of measurement and strategic management, which has been implemented in the public sector. In the state of Mato Grosso, that model has been used in 23 municipalities by an initiative of Tribunal de Contas do Estado - TCE, through the Integrated Institutional Development Program (Programa de Desenvolvimento Institucional Integrado - PDI, in Portuguese). The general objective of the study was to describe how the BSC tool has contributed to the
\end{abstract}


improvement of municipal public management. More especifically: a) to identify the important factors in the correct implementation of the BSC tool in the literature; b) to identify how the tool is being applied in the municipalities of Mato Grosso do Sul; and c) to describe how the tool is operationalized in the perception of the managers of municipal planning control. The methodology is characterized as basic qualitative, inductive type with descriptive character, through the application of a structured questionnaire. The main result was the alignment of the strategic planning model of the municipalities with the BSC model in their respective dimensions. Finally, it was found that in the perception of the respondents the tool was successfully implemented by the municipalities, favoring the improvement of municipal public management.

Keywords: Balanced Scorecard, Alignment, Public Management.

Submetido: 05/01/2021

Aceito: 13/03/2021

Publicado: 30/04/2021

\section{CONSIDERAÇÕES INICIAIS}

O atual modelo de gestão pública existente no Brasil provém das diversas modificações a respeito das atividades de natureza administrativa, que são marcadas pelas relações entre Estado e Sociedade, onde encontram-se inseridos grupos de pessoas que são comandadas por um governante.

Dentro desta perspectiva, as primeiras marcas de uma administração pública são encontradas no período colonial, mais especificamente na administração pública patrimonial. Diante da necessidade de se distinguir público e privado e da determinação de um método de governo que fosse mais racional e eficiente e que substituísse os regimes autoritários, surge por volta da década de 30, a administração pública burocrática (HELAL; DIEGUES, 2009). Em meio a um cenário político conturbado nas décadas de 80 e 90, surge a administração pública gerencial, como um meio de enfrentar a crise sofrida e para que se tornasse possível a transformação dos serviços prestados pelo governo, tornando-os mais eficientes, sendo assim uma gestão orientada para o cidadão e para o alcance de resultados (ANTONIO et al., 2016).

Portanto, a administração pública gerencial trouxe a descentralização do poder, as noções de eficiência e eficácia e os primeiros conceitos acerca de avaliação de desempenho dos colaboradores. E como forma de medir o desempenho das organizações, tanto públicas quanto privadas, são usados alguns recursos como os indicadores de desempenho, que são um conjunto de medidas que focam a respeito dos aspectos do desempenho organizacional (PARMENTER, 2007).

Considerando o histórico da administração pública no Brasil, um importante marco a ser mencionado foi a criação do Programa Nacional de Gestão Pública e Desburocratização - GesPública, instituído pelo decreto 5.378/2005, com o objetivo de implantar soluções que permitissem o aperfeiçoamento dos sistemas de gestão das organizações públicas, possuindo em seus documentos norteadores um manual de modelo de excelência em gestão pública que abrange orientações para que a administração pública alcance a excelência.

Para que se possa implantar a excelência nas organizações públicas ou utilizar os indicadores de desempenho antes de mais nada é necessário um planejamento 
estratégico, que segundo Oliveira (2010, p. 17), “é o processo administrativo que proporciona sustentação metodológica para se estabelecer a melhor direção a ser seguida pela empresa" ou seja o planejamento é um processo de definição das melhores estratégias a serem utilizadas para se chegar a objetivos predeterminados.

Uma das ferramentas mais utilizadas para medir o desempenho da organizações é o Balanced Scorecard (BSC), sendo uma metodologia que de acordo com Kaplan e Norton (1997 apud HERRERO FILHO, 2005, p. 25) "traduz a missão e a visão das empresas num conjunto abrangente de medidas de desempenho que serve de base para um sistema de medição e gestão estratégica". Essa ferramenta foi inicialmente pensada para o setor privado, porém aos poucos tem sido implementada no setor público, como forma de contribuir na inserção de uma cultura de medição de desempenho e de auxiliar no aumento da capacidade da organização de reagir as demandas externas (GHELMAN; COSTA, 2006).

No estado de Mato Grosso, esse modelo passou a ser utilizado em alguns municípios a partir de uma iniciativa do Tribunal de Contas do Estado - TCE, por meio do Programa de Desenvolvimento Institucional Integrado- PDI desenvolvido pela Secretaria de Apoio às Unidades Gestoras - SAUG.

Tendo em vista o modelo de administração pública onde prevalece a cultura da falta de planejamento e a experiência de utilização da ferramenta BSC nas prefeituras de 23 municípios do estado de Mato Grosso, estabeleceu-se o seguinte problema de pesquisa: Como está sendo aplicada a ferramenta BSC nos municípios que participam do programa PDI em MT?

O Objetivo geral foi descrever como a ferramenta Balanced Scorecard - BSC tem contribuído para o aprimoramento da gestão pública municipal. De maneira específica buscou-se a) identificar os fatores importantes na correta implementação da ferramenta BSC na literatura; b) identificar como a ferramenta está sendo aplicada nos municípios de MT; e por fim, c) descrever como a ferramenta é operacionalizada na percepção dos gestores do controle do planejamento municipal.

Do ponto de vista social, uma melhor gestão possibilita um aumento da qualidade na prestação dos serviços públicos, pois conforme mencionado por Rua (2004, p. 4), os indicadores possibilitam a "formação de um serviço público eficaz, eficiente, flexível, transparente, altamente capacitado, profissionalizado e comprometido com o bem-estar da sociedade". Do ponto de vista econômico o uso do planejamento estratégico proporciona uma redução de custos para as organizações, sendo elas privadas ou públicas, uma vez que busca a otimização dos recursos (SOUZA, 2010). Por fim, na perspectiva política, pois de certa forma, promove os gestores, uma vez que resultados positivos obtidos a partir da implementação do planejamento estratégico eleva a aprovação da gestão responsável que pode proporcionar a longevidade de sua atuação política.

\section{Planejamento estratégico e o BSC}

O planejamento é uma ferramenta administrativa que serve como apoio para a tomada de decisões relacionadas com o alcance dos objetivos almejados, ou seja, está ligado diretamente com as relações futuras. Pereira (2010, p. 44) define planejamento como o "processo formalizado para gerar resultados a partir de um sistema integrado de decisões". É também a definição e adoção de objetivos, estratégias e ações orientadas pelo ambiente, tanto interno quanto externo, que serão utilizadas no processo de tomada de decisões (GULLO, 2016). 
Dessa forma para um funcionamento eficiente e eficaz das organizações, se faz necessário a implantação de um planejamento estratégico, que pode ser definido como um processo de formulação de estratégias elaboradas com o objetivo de alcançar uma visão de futuro (SILVA; MÁRIO, 2015), sendo as estratégias um conjunto integrado de ações definidas para explorar competências e obter vantagem competitiva (HITT; IRELAND; HOSKISSON, 2011). A função do planejamento estratégico segundo SILVA (2013) é Estabelecer processos, atitudes e técnicas administrativas, a fim de construir um ambiente sustentável capaz de avaliar corretamente os resultados futuros das decisões do presente.

Na visão de Ribas, Facini e Teixeira (2014), o processo de planejamento passa por 3 etapas principais, sendo elas a definição de objetivos, definição dos meios de execução e definição dos meios de controle. Na primeira etapa são estabelecidos os objetivos gerais, ou seja, aonde se quer chegar através do planejamento, na segunda etapa são determinados os meios para se alcançar o objetivo e por fim na terceira etapa são definidos os meios de controle para verificar se os objetivos estão sendo cumpridos.

No entanto, apenas estas etapas não bastam para um planejamento estratégico eficaz, de modo que, se faz necessário o uso da administração estratégica, definida como um "processo contínuo e interativo que visa manter uma organização como um conjunto apropriadamente integrado a seu ambiente" (CERTO et al., 2005, p. 3) ou ainda como "todo o processo de escolha, planejamento, execução e avaliação de escolhas" (SERTEK; GUINDANI; MARTINS, 2007, p. 42).

Os conceitos de planejamento estratégico e administração estratégica são distintos, porém funcionam em conjunto e se complementam. Enquanto o planejamento estratégico trata do processo de tomada de decisões, a administração estratégica se preocupa em obter resultados, como aumento de lucratividade ou um maior comprometimento dos membros da organização a cumprir metas e objetivos. As etapas básicas da administração estratégica incluem análise do ambiente, estabelecimento da diretriz organizacional, formulação da estratégia, implementação da estratégia e controle estratégico (CERTO et al., 2005).

O primeiro passo da administração estratégica é a análise do ambiente, através da avaliação do ambiente externo e interno para identificação de possíveis oportunidades e riscos que possam influenciar o alcance de metas da empresa (CERTO et al., 2005). Um método muito utilizado nessa etapa de diagnóstico do ambiente é a Matriz Swot (Strenght, Weaknesses, Opportunities e Threats), com o objetivo de reunir os pontos fortes, pontos fracos, oportunidades, ameaças e relacioná-los (PEREIRA, 2010).

No estabelecimento da diretriz organizacional são definidos a missão e os objetivos, sendo a missão a razão de ser da empresa e os objetivos as metas que ela possui. Em seguida são formuladas as estratégias de ação que a organização em questão precisa realizar para atingir seus objetivos. No entanto não basta apenas definir as estratégias, elas precisam ser colocadas em prática para que a empresa possa obter os resultados desejados.

A etapa final é a de controle estratégico, Ribas, Facini e Teixeira (2014) definem que o controle possui a finalidade de avaliar as etapas anteriores do processo de desenvolvimento. Já o controle estratégico é um tipo específico de controle organizacional que se preocupa em monitorar e avaliar o processo de administração estratégica afim de melhorá-lo, para isso são realizadas três etapas distintas, a primeira que mede o desempenho organizacional, a segunda que compara o desempenho 
organizacional com os objetivos estabelecidos e pôr fim a etapa que determina a ação corretiva (CERTO et al., 2005).

Uma das ferramentas gerenciais mais utilizadas para implantar um sistema de planejamento estratégico e controle organizacional é o Balanced Scorecard, que cria mapas estratégicos a partir de quatro perspectivas: perspectiva financeira, perspectiva dos clientes, perspectiva dos processos internos e perspectiva do aprendizado e crescimento (PEREIRA, 2010).

O Balanced Scorecard (BSC) é um "sistema de gestão que traduz a estratégia de uma empresa em objetivos, medidas, metas e iniciativas de fácil entendimento pelos participantes da organização" (HERRERO FILHO, 2005, p. 26). Desenvolvido por Kaplan e Norton em 1992, com o foco principal de traduzir a visão da organização em ações concretas, por meio do estabelecimento de metas e indicadores de desempenho (COSTA, 2003). É um sistema que controla desde a identificação das necessidades até a motivação das melhorias dos processos e produtos, gerando um ambiente apropriado para o alinhamento estratégico organizacional (FELIX; FELIX; TIMÓTEO, 2011). O BSC ainda possibilita uma tomada de decisões com base no alinhamento das medidas de desempenho com as metas e estratégias da organização, portanto quanto maior o grau de alinhamento, maior a sintonia entre os objetivos estratégicos (LUCIANETTI; BATTISTA; KOUFTEROS, 2019).

Sua metodologia propõe a tradução da missão e visão em objetivos e medidas através de um mapa estratégico, classificados sob a ótica de quatro perspectivas: financeira, do cliente, dos processos internos e de aprendizado e crescimento, conforme a Figura 1 (KAPLAN; NORTON, 1997). A perspectiva financeira indica se a estratégia de uma empresa está contribuindo para a melhorias dos resultados financeiros, assim os objetivos estão relacionados a lucratividade (KAPLAN; NORTON, 1997).

Figura 1. Perspectivas do BSC

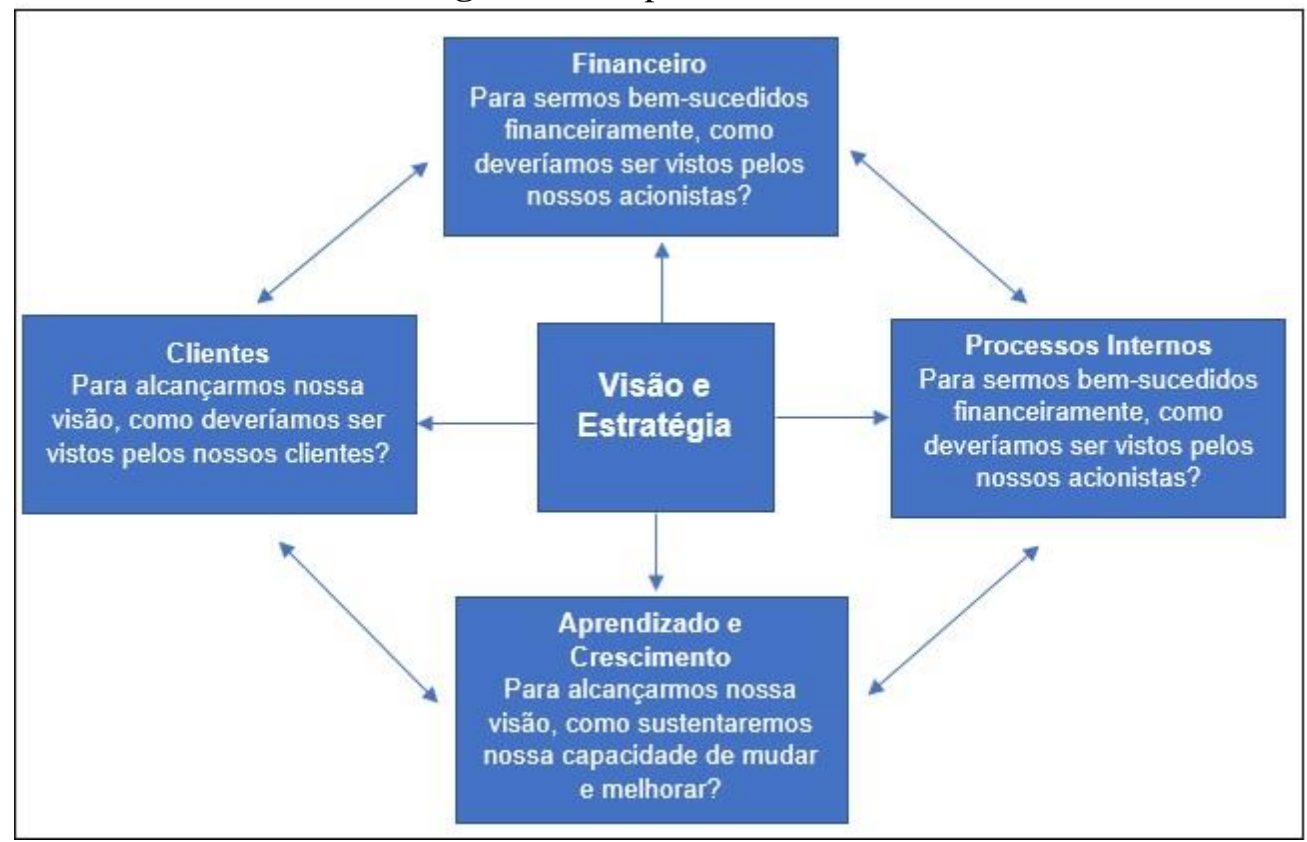

Fonte: Kaplan e Norton (1997)

A perspectiva dos clientes avalia se a proposta de valor da empresa para os clientes está produzindo resultados positivos relacionados a satisfação do cliente, 
conquista de novos clientes, retenção de clientes e outros. Na perspectiva de processos internos, são identificados os processos críticos em que a empresa precisa alcançar a excelência para que possa impactar na satisfação dos clientes e nos objetivos financeiros. E a perspectiva da aprendizagem e do conhecimento identifica se a aprendizagem, a conquista de novos conhecimentos e o domínio de competências são viáveis para o desempenho das perspectivas anteriores (HERRERO FILHO, 2005).

Kaplan e Norton (1997) dizem que as medidas do BSC devem ser usadas de diferentes maneiras, como para articulação de estratégias, comunicação de estratégias e suporte no alinhamento de iniciativas individuais, organizacionais $\mathrm{e}$ interdepartamentais, os autores ainda afirmam que o Balanced Scorecard não deve ser utilizado somente como um sistema de controle, mas sim como um sistema de comunicação, informação e aprendizado.

No ambiente competitivo onde estão inseridas as organizações, um dos desafios mais complexos para os gestores é o alinhamento entre as metas individuais e as metas estratégicas da empresa (HERRERO FILHO, 2005). É importante destacar que o suporte da alta administração é indispensável para a eficácia do $\mathrm{BSC}$, onde o comprometimento e a liderança desta gestão são fatores chaves para um bom desempenho (LUCIANETTI; BATTISTA; KOUFTEROS, 2019).

Contudo a comunicação das estratégias a todos os níveis da organização, desde a alta administração até a gestão de base, são uma maneira de garantir que todos estejam alinhados ao cumprimento do objetivo final da estratégia, sendo importante também que toda organização participe da criação das estratégias e não somente a alta administração, pois dessa forma será possível uma combinação de diversos tipos de conhecimento que contribuíram para o êxito do BSC, evitando que ele seja comprometido (NASCIMENTO JÚNIOR; SILVA; MOTA, 2014).

No entanto para que as pessoas se sintam motivadas a estarem envolvidas com as mudanças estratégicas elas precisam identificar oportunidades de recompensas e crescimento pessoal (HERRERO FILHO, 2005), porém no BSC a falta de esquemas de incentivo tem sido considerado uma barreira a eficácia do sistema, portanto os incentivos no BSC merecem uma atenção maior, visto que é uma ferramenta que pode ser reforçada quando as medidas de desempenho estão alinhadas com o esquema de recompensas (LUCIANETTI; BATTISTA; KOUFTEROS, 2019).

Tendo em vista que o planejamento estratégico é uma ferramenta que auxilia o processo de tomada de decisões para o alcance de objetivos futuros, a próxima seção irá abordar a respeito da utilização do planejamento estratégico na Administração Pública.

\section{Planejamento estratégico na administração pública}

É perceptível a importância dada ao planejamento estratégico nos setores públicos e privados, visto que empresas e organizações públicas tem passado por mudanças nos ambientes econômico, social, tecnológico e político, dessa forma tornouse necessário à adaptação as mudanças para que as organizações possam evoluir diante dessa conjuntura (PEREIRA, 2007).

Sendo assim, a prática do planejamento estratégico pode ser considerada essencial na gestão das organizações, pois traz inúmeros benefícios como a conquista de uma maior visão de futuro, um melhor preparo para reagir diante das dificuldades, identificação de oportunidades e integração de áreas e pessoas na execução dos objetivos (REBELO; ALMEIDA, 2008). 
É notável que com o passar dos anos os cidadãos ficam cada vez mais exigentes em relação à prestação dos serviços públicos. Portanto na administração pública o planejamento pode ser considerado o ponto de partida da eficiência e da eficácia, uma vez que ele determinará os rumos da gestão, o que reflete na qualidade dos serviços prestados (SILVA et al., 2013).

A administração pública contempla todo "conjunto de ideias, atitudes, normas, processos, instituições e outras formas de conduta humana que trabalham no sentido de exercer a autoridade política a fim de atender os interesses públicos" (SILVA et al., 2013, p. 91). No processo de gestão da administração pública o maior desafio encontrado é conseguir transformar os processos burocráticos em processos mais flexíveis e isso pode ser superado através do uso de instrumentos de gestão como o planejamento estratégico.

Antes de se mencionar sobre as etapas de implantação do planejamento estratégico em organizações públicas é fundamental a compreensão acerca dos conceitos de eficiência, eficácia e efetividade, pois são essenciais no contexto do setor público. Conforme Chiavenato (2008), a eficácia está relacionada à realização de algo de maneira certa, estando vinculada ao alcance de objetivos, enquanto a eficiência consiste na forma pela qual realiza-se algo, ou seja, o caminho percorrido para se atingir o objetivo de maneira que seja consumido os recursos necessários de forma racional. E a efetividade "preocupa-se em identificar em que medida os resultados de uma ação trazem benefício à população" (REBELO; ALMEIDA, 2008).

Não há um consenso ou uma definição exata das etapas a serem seguidas na elaboração do planejamento estratégico, pois como é uma ferramenta flexível, pode ser adaptada de acordo com a necessidade do ambiente a ser inserida. Entretanto, Pereira (2007) define que a implementação do planejamento estratégico em uma organização pública consiste na realização dos seguintes passos: Sensibilização e mobilização de atores, diagnóstico prévio, oficinas, implementação, monitoramento e revisão. No primeiro passo ocorre a definição de quem planeja, a fase de diagnóstico visa identificar os problemas existentes, as oficinas são uma série de atividades desenvolvidas, como análise do ambiente e definição de estratégias.

No passo de implementação são realizados procedimentos específicos para agregar as ações estabelecidas nas oficinas no dia a dia da organização, já o monitoramento buscar identificar os indicadores de resultados, indicadores de processo e indicadores contextuais. E por último na revisão, conforme os dados e informações obtidas no monitoramento, é realizada uma atualização do planejamento, com vista ao aperfeiçoamento do sistema (PEREIRA, 2007).

Vale ressaltar que a maior ênfase no planejamento no setor público se deu a partir da Constituição Federal de 1988, onde o orçamento público foi aliado ao planejamento, juntamente com a Carta Magna que apontou a integração de ferramentas de planejamento no setor público, destacando assim o Plano Plurianual (PPA), a Lei de Diretrizes Orçamentárias (LDO) e a Lei Orçamentária Anual (LOA) (SILVA et al., 2013). Esses instrumentos, no entanto, dão ênfase ao planejamento financeiro, sem considerar o aspecto tático e operacional.

Como já mencionado as organizações públicas precisam adaptar-se às mudanças sofridas nos ambientes nos quais elas estão inseridas e para além disso devem também saber lidar com outros fatores, como o crescimento dos gastos do setor público, o atendimento da necessidade da população de serviços de qualidade, entre outros desafios que forçam os gestores a buscarem a eficiência e eficácia nas atividades 
exercidas, conforme afirmam Kaplan e Norton (1997, p.189) "no caso das empresas públicas e instituições sem fins lucrativos, o sucesso deve ser medido pelo grau de eficácia e eficiência com que essas organizações atendem às necessidades de seus participantes".

Assim para que se possa ser medido esses índices se faz necessário a utilização de instrumentos como o Balanced Scorecard para avaliação e monitoramento de desempenho na gestão pública (FARIA; FERNANDES; GUIMARÃES, 2008). O BSC foi inicialmente utilizado no setor privado, mas dado a demanda tem sido implantado no setor público, porém considerando as diferenças existentes entre os setores, onde um tem como foco o lucro e outro o foco na satisfação do cidadão, a ferramenta precisa de adaptações para que tenha sucesso em sua aplicação (BEMFICA; CALLADO, 2016).

No que se refere às perspectivas do BSC, a que está ligada ao cidadão é a perspectiva de clientes, que "permite que as empresas alinhem suas medidas essenciais de resultados relacionados ao clientes - satisfação, fidelidade, retenção, captação e lucratividade - com segmentos específicos de clientes e mercados" (KAPLAN; NORTON, 1997, p. 67), permite também a criação de uma proposta de valor capaz de atender às necessidades do cliente, que no caso do setor público é o cidadão, qual possui necessidades que precisam ser supridas para estar satisfeito. Portanto essa é uma perspectiva essencial no BSC da gestão pública.

De acordo com Silva (2009) uma adaptação que deve ser realizada diz respeito à alteração da posição da perspectiva financeira, que deixa de aparecer no topo para dar lugar à perspectiva de clientes, o que é justificado pelo fato do objetivo das organizações públicas serem distintas das privadas. Apesar das facilidades de adaptação da ferramenta, existem algumas complicações na efetiva implementação do BSC na gestão pública, como a dificuldade em definir indicadores que monitorem $o$ desempenho da entidade (PEDRO, 2004), a resistência a mudanças (SILVA, 2009), a complexidade e o tempo ligado ao desenvolvimento e revisões periódicas dos indicadores (LUCIANETTI; BATTISTA; KOUFTEROS, 2019) e ainda a maior dificuldade segundo Kaplan e Norton (2001) está relacionada a definição de estratégias.

No que tange ao desenvolvimento e revisões periódicas do BSC, os autores Lucianetti, Battista e Koufteros (2019) afirmam que as atualizações da ferramenta são mais importantes até que o processo de desenvolvimento e implementação, sendo assim se faz necessária a revisão regular dos resultados, podendo ser de 3 a 4 vezes ao ano ou até mesmo uma vez por mês, conforme a necessidade da organização.

\section{Indicadores de desempenho}

Um conceito de indicadores é dado por Helou Filho e Otani (2006, p. 9) em que os indicadores "são funções que permitem obter dado ou informação numérica sobre as medidas relacionadas a um sistema, um processo, um produto ou uma grandeza, sendo utilizados para acompanhar e melhorar os resultados do objeto de estudo ao longo do tempo".

Os indicadores de desempenho podem ser considerados uma ferramenta de gestão capaz de analisar os resultados dos processos realizados dentro das organizações de forma mais direcionada, levando em consideração que o desempenho é algo único e específico este pode ser definido como " a soma de esforços em direção a resultados a serem obtidos" (LANGWINSKI, 2013, p. 24).

Souza (2010, p.52) diz que: 
Um sistema de indicadores de desempenho bem planejado e estruturado oferece a possibilidade de um grau maior de compromisso com resultados, mediante a determinação de metas de desempenho que expressem os sucessos esperados em termos de quantidade e qualidade dos serviços prestados e da efetividade e eficiência com que são oferecidos.

Dessa forma a função dos indicadores de desempenho é fornecer dados para que as organizações possam verificar se as melhorias implementadas produziram resultados positivos, como as atividades estão sendo realizadas, se os objetivos estão sendo atingidos e que aspectos necessitam de mudanças (HELOU FILHO; OTANI, 2006).

Quanto a classificação dos indicadores de desempenho Morissete (1977 apud ZANIN et al., 2015) os classifica em indicadores financeiros e não financeiros, sendo o indicador financeiro uma medida quantitativa que é expressa em valores monetários como o lucro, enquanto os indicadores não financeiros também são uma medida quantitativa mas que não é expressa em valor monetário, como a participação no mercado.

Levando em consideração que os indicadores de desempenho são uma ferramenta de grande importância para a gestão empresarial e que diversos são os modelos de indicadores, pode-se mencionar que "uma das mais relevantes contribuições para o processo de gestão estratégica mediante o uso de indicadores deve-se ao Balanced Scorecard, que complementa os indicadores financeiros" (ARAUJO, 2001, p.100).

\section{Indicadores de desempenho na administração pública}

Dado o atual modelo de administração pública gerencial orientada para a busca de resultados e com foco no cidadão, é possível notar que o governo vem realizando diversos esforços com vista a modernização da gestão pública e consequente melhoria da qualidade dos serviços prestados, com uma visão voltada para "formação de um serviço público eficaz, eficiente, flexível, transparente, altamente capacitado, profissionalizado e comprometido com o bem-estar da sociedade" (RUA, 2004, p. 4).

Pode-se mencionar como um desses esforços a utilização de indicadores de desempenho, que "são instrumentos que permitem identificar e medir situações de uma realidade dada, pois traduzem de forma mensurável aspectos que se pretende aferir ou monitorar" (JESUS et al., 2014,p.6). No âmbito da gestão pública os indicadores "são instrumentos que contribuem para identificar e medir aspectos relacionados a um determinado fenômeno decorrente da ação ou da omissão do Estado" (MINISTÉRIO DO PLANEJAMENTO, 2012, p.16).

Dessa forma o principal objetivo dos indicadores na administração pública é o de auxílio aos gestores públicos, principalmente no que se refere ao processo de tomada de decisões, pois estas pessoas passarão a pensar de maneira estratégica e deixarão de realizar decisões apenas pela intuição, uma vez que as decisões serão tomadas a partir das informações obtidas por meio dos indicadores.

O desempenho no âmbito governamental está ligado aos conceitos de eficiência e eficácia, nesse contexto a eficiência é a capacidade de realizar uma atividade com o menor custo e a eficácia verifica se os objetivos anteriormente definidos para realização da atividade estão sendo atendidos, além de recentemente terem sido incorporados as dimensões do desempenho orçamentário, foco e qualidade na prestação de serviços (BONNEFOY; ARMIJO, 2005). 
Conforme Bonnefoy e Armijo (2005), os indicadores ainda possuem duas funções, a função descritiva e função avaliativa. A primeira se refere ao fornecimento de informações sobre o estado de um programa público, enquanto a função avaliativa acrescenta valor a essa informação, com base no objetivo de desempenho de um determinado programa público, se ele é ou não adequado. Os autores ainda classificam os indicadores de desempenho no âmbito público em indicadores que fornecem informações sobre o ponto de vista do desempenho público na geração de insumos, processos e atividades, produtos e resultados finais e indicadores ligados ao desempenho dessas dimensões na eficiência, eficácia, qualidade e economia.

Rua (2004) ainda classifica os indicadores em indicadores estratégicos, que refletem o desempenho em relação aos objetivos estratégicos da organização, indicadores de processo, que medem a eficiência e a eficácia do processo e neles estão inclusos os indicadores de qualidade, produtividade e capacidade e indicadores de projetos, que acompanham e avaliam a execução de projetos, além de definir que para os indicadores se tornem viáveis e práticos precisam de alguns atributos como adaptabilidade, representatividade, economia e estabilidade.

Sendo assim, "os indicadores podem e devem ser utilizados para melhorar a gestão interna já que permitem o controle das atividades, a motivação dos colaboradores e a identificação de problemas e possíveis soluções" (JESUS et al., 2014, p.7).

\section{PROCEDIMENTOS METODOLÓGICOS}

Como se deseja conhecer a visão de um determinado público alvo, sobre um tema específico com base na revisão bibliográfica e nas respostas obtidas através do instrumento de pesquisa aplicado, a pesquisa é definida como de natureza qualitativa de carácter indutivo com objetivo descritivo.

O método indutivo parte da observação de fatos ou fenômenos cujos motivos se deseja conhecer, comparando as relações existentes para assim poder realizar a generalização (GIL, 2008). E descritiva pois "observa, registra, analisa e ordena dados, sem manipulá-los, isto é, sem interferência do pesquisador" (PRODANOV; FREITAS, 2013, p. 52). Além de ser um estudo transversal, pois os dados foram coletados em um determinado ponto no tempo.

A população alvo são os gestores das prefeituras de 23 municípios do estado de Mato Grosso que participam do Programa de Desenvolvimento Institucional Integrado, do Tribunal de Contas de Mato Grosso. A amostra escolhida foi a não-probabilística por conveniência, pois dentro da população foram selecionados os indivíduos que tiveram disponibilidade e que fizessem uso da ferramenta $\mathrm{BSC}$, conforme a descrição realizada por Hair Junior et al. (2005, p. 247) em que "a amostra por conveniência envolve a seleção de elementos de amostra que estejam mais disponíveis para tomar parte no estudo e que podem oferecer as informações necessárias".

O instrumento de coleta de dados utilizado foi um questionário estruturado que é uma técnica de investigação que conforme Gil (2008, p.121) é constituída por um "conjunto de questões que são submetidas a pessoas com o propósito de obter informações sobre conhecimentos, crenças, sentimentos, valores, interesses, expectativas (...) etc.". Esse instrumento foi escolhido pela praticidade na coleta e organização dos dados junto a um público mais heterogêneo e geograficamente disperso. 
O questionário aplicado foi retirado dos estudos de Lucianetti, Battista e Koufteros (2019), contendo 19 perguntas do tipo fechadas, que possibilita ao respondente a escolha de apenas uma alternativa entre as diversas apresentadas e como forma de mensuração dessas questões foram utilizadas afirmativas do tipo discordo totalmente à concordo totalmente, dentro da escala de Likert que "consiste em tomar um construto e desenvolver um conjunto de afirmações relacionadas à sua definição, para as quais os respondentes emitirão seu grau de concordância" (SILVA JÚNIOR; COSTA, 2014, p. 5). A aplicação dos questionários se deu via formulário eletrônico na plataforma Google Docs. O link do formulário foi disponibilizado via e-mail e WhatsApp para os respondentes dos 23 municípios. No entanto, o retorno foi muito pequeno, apenas 14 questionários respondidos, além disso, foram invalidados 2 questionários, o que resulta numa amostra considerada muito pequena de apenas 12 gestores, no entanto, é uma amostra representativa de mais de 50\% dos municípios que participam do programa.

Para análise dos dados foi adotada uma abordagem de pesquisa qualitativa básica, que "envolve a obtenção de dados descritivos sobre pessoas, lugares e processos interativos pelo contato direto do pesquisador com a situação estudada procurando compreender os fenômenos segundo a perspectiva dos sujeitos" (GODOY, 1995, p. 58), com a intenção de descrever a percepção e vivência dos gestores em relação a aplicação do modelo do BSC na realidade organizacional de suas prefeituras. De forma complementar, buscou-se comparar essa percepção dos gestores com as indicações da literatura para uma melhor implementação da metodologia do BSC.

Entre as limitações dessa pesquisa destaca-se o uso do questionário estruturado como instrumento de coleta de dados, uma vez que limita drasticamente a capacidade de captar as percepções e impressões pessoais dos respondentes. Outra limitação foi o baixo número de respondentes.

\section{Caracterização do objeto de estudo}

O Tribunal de Contas do Estado de Mato Grosso é o órgão responsável pela fiscalização e controle das contas públicas do Estado, criado em 1953 a partir da lei constitucional $\mathrm{n}^{\circ} .02$ que extinguiu a comissão legislativa, que antes era responsável por gerir as contas públicas e cria o Tribunal de Contas do Estado de Mato Grosso (TCEMT). Algumas das atividades exercidas pelo TCE-MT se referem a emissão de parecer sobre as contas prestadas anualmente pelo governador e pelo prefeitos municipais, julgamento das contas dos responsáveis por dinheiro, bens e valores públicos da administração direta e indireta, fiscalização do cumprimento de normas, entre outros (TRIBUNAL DE CONTAS DE MATO GROSSO, [s.d.]).

No ano de 2012 o Tribunal de Contas instituiu o Programa de Desenvolvimento Institucional Integrado (PDI) com o objetivo de contribuir no aprimoramento da eficiência nos serviços públicos, através de um modelo de administração pública voltada para os resultados. O programa possui o foco de estabelecer ações transversais capazes de promover o desenvolvimento integrado e permanente do TCE-MT e de todas as instituições públicas por ela fiscalizadas (MATO GROSSO, 2013).

O PDI pretende inserir a cultura do planejamento, da transparência, da eficiência e da inovação na administração pública e na sociedade de Mato Grosso, para isso foram criados cinco projetos: Apoio ao planejamento estratégico; incentivo ao acesso à informação e à consciência cidadã; orientação por meio de cursos presenciais e a 
distância; controle gerencial utilizando o sistema Geo-Obras e modernização institucional. Posteriormente foi incluído o projeto TCEndo a cidadania.

$\mathrm{O}$ projeto 1 de apoio ao planejamento estratégico, possui o objetivo de disseminar a cultura do planejamento estratégico e da administração pública gerencial nas unidades fiscalizadas pelo TCE-MT, isso ocorre por meio de fornecimento de ferramentas tecnológicas e orientação técnica das equipes (MATO GROSSO, 2013). Atualmente o PDI está sendo desenvolvido nos seguintes municípios: Água Boa, Alta Floresta, Cáceres, Campo Verde, Chapada dos Guimarães, Confresa, Cuiabá, Diamantino, Itiquira, Jaciara, Juscimeira, Juína, Lucas do Rio Verde, Nortelândia, Primavera do Leste, Querência, Rondonópolis, São Félix do Araguaia, São José dos Quatro Marcos, Sapezal, Sinop, Tangará da Serra, Tapurah e Várzea Grande.

\section{APRESENTAÇÃO E ANÁLISE DOS RESULTADOS}

Dos municípios integrantes do Programa de Desenvolvimento Institucional Integrado, participaram dessa pesquisa, apenas os gestores dos municípios de Água Boa, Campo Verde, Confresa, Cuiabá, Jaciara, Juína, Nortelândia, Querência, São Félix do Araguaia, São José do Quatro Marcos, Sinop e Várzea Grande, o que compreende apenas metade da amostra pretendida.

Na busca de avaliar o nível de desenvolvimento que se encontra a ferramenta BSC na gestão pública dos municípios de Mato Grosso participantes da pesquisa, identificou-se que 11 de 12 respondentes descreveram que o nível de desenvolvimento do BSC na sua organização é de uma estrutura multidimensional, o qual combina medidas estratégicas financeiras e não financeiras, que descreve a estratégia usando relações de causa e efeito e que ainda implementa a estratégia definindo objetivos, planos de ação, resultados e incentivos com o BSC.

Com base na literatura esse resultado indica que os municípios estão alinhados com a recomendação de Kaplan e Norton (1997) sobre o equilíbrio entre medidas financeiras e não financeiras no BSC, e ainda, corrobora com Bemfica e Callado (2016) que descrevem o BSC como uma ferramenta composta por uma estrutura de objetivos e metas que interagem dentro de uma lógica de causa e efeito, possibilitando a medição do desempenho organizacional sob quatro perspectivas do mapa estratégico.

Quanto à aplicação da ferramenta por meio da utilização do mapa estratégico em associação com as 4 perspectivas do BSC, novamente 11 de 12 respondentes afirmam utilizar esse modelo padrão de perspectivas, o que está de acordo com a definição do BSC de Pereira (2010), que descreve a implantação do planejamento estratégico e controle organizacional através de mapas estratégicos a partir de quatro perspectivas.

No que diz respeito à comunicação das estratégias definidas no BSC aos níveis organizacionais, identificou-se uma tendência maior de respostas positivas concordando com a ampla comunicação para os níveis de alta gestão, gestão intermediária e gestão de base, ou seja, os gestores acreditam que todos ou pelo menos a maioria dos indivíduos da organização, independente de seu nível organizacional conhecem as estratégias do BSC. Esse resultado vai ao encontro com o mencionado pelos autores Nascimento Júnior, Silva e Mota (2014) em que a comunicação da estratégia a todos os níveis da organização e não somente a alta administração pode assegurar que todos os colaboradores estejam envolvidos.

Essa percepção pode ser sustentada pelo fato do TCE-MT fornecer de forma gratuita a plataforma do Gerenciamento do Planejamento Estratégico - GPE, que 
possibilita o uso de uma ferramenta para monitoramento e comunicação do desempenho da organização e suas partes em relação ao planejamento estratégico (TRIBUNAL DE CONTAS DE MATO GROSSO, 2018).

No que se refere ao grau de alinhamento interno entre os objetivos estratégicos e as medidas de desempenho incluídas no BSC observou-se uma tendência positiva para o alinhamento total, pois 10 de 12 participantes afirmaram que os objetivos da sua organização estão totalmente alinhados com as medidas de desempenho do BSC, o que demonstra a sintonia entre os objetivos estratégicos, conforme mencionado por Lucianetti, Battista e Koufteros (2019).

Nos municípios estudados, verificou-se que em 58,3\% deles, os gestores concordaram que os esquemas de incentivos para os funcionários dependem do desempenho da equipe e $66,6 \%$ das respostas apontam que os incentivos para os funcionários dependem de medidas de desempenho não financeiras. Quanto aos incentivos para os funcionários dependerem do sistema BSC, as respostas apresentaram uma tendência mais negativa, o que pode significar que nas organizações em questão as recompensas não dependem exclusivamente do BSC.

Como se trata de organizações públicas geralmente as recompensas dos funcionários são estáveis e estão ligadas ao cargo para o qual prestaram o concurso público (FRANCO et al., 2017). Se existir uma ligação entre o desempenho e os resultados é uma mudança que aos poucos o setor público vem buscando implementar. Sendo assim, se a percepção dos respondentes está correta, os municípios estudados adotam uma gestão de pessoas mais alinhada com o padrão de recompensas e remuneração, o que destoa do que é identificado por Franco et al. (2017).

Além dos incentivos dependerem de medidas não financeiras de desempenho foi possível verificar uma tendência positiva de respostas que demostram que os incentivos dos colaboradores também dependem de medidas de desempenho financeiras. E por fim no que tange à esta análise, a respeito dos incentivos do BSC, cerca de $75 \%$ dos gestores dizem que eles dependem do desempenho individual do funcionário, ou seja, a necessidade do desempenho individual é maior do que a do desempenho da equipe. Esses resultados, indicam que os incentivos não seriam um fator considerado como uma barreira à eficácia do sistema, o que destoa com o mencionado anteriormente por Lucianetti, Battista e Koufteros (2019), e não aparenta ser a realidade das organizações públicas brasileiras.

Sabe-se que o modelo do BSC criado por Kaplan e Norton (1997) abrange a perspectiva financeira, perspectiva de clientes, perspectiva de processos internos e perspectiva de aprendizado e crescimento, assim os gestores tiveram que atribuir uma pontuação a estas perspectivas de acordo com o nível de sua importância para a organização, com isso foi possível ver um certo padrão nas respostas, pois a perspectiva financeira apresentou as maiores respostas dos gestores entre 25 a 30 pontos, a perspectiva de clientes teve uma maior oscilação, apresentando respostas entre 20 a 30 pontos e as perspectivas de processos internos e aprendizado e crescimento, obtiveram uma distribuição mais uniforme em torno dos 25 pontos.

Esses resultados indicam que na percepção dos respondentes as quatro perspectivas sugeridas no BSC têm o mesmo grau de importância para as prefeituras. Foi dada aos participantes a possibilidade de incluir outra perspectiva além daquelas propostas pelo BSC, apenas duas respostas mencionaram outras perspectivas, uma mencionou convênios e outra parcerias, dimensões que aparente estão relacionadas e que demandam uma investigação mais profunda em estudos futuros. 
Dentro dessas perspectivas estão inseridas as medidas de desempenho ou indicadores de desempenho, dessa forma os gestores foram questionados acerca do número de medidas de desempenho utilizadas pelos seus municípios, as respostas podem ser visualizadas no Gráfico 1.

Gráfico 1. Número de medidas de desempenho utilizadas pelos municípios

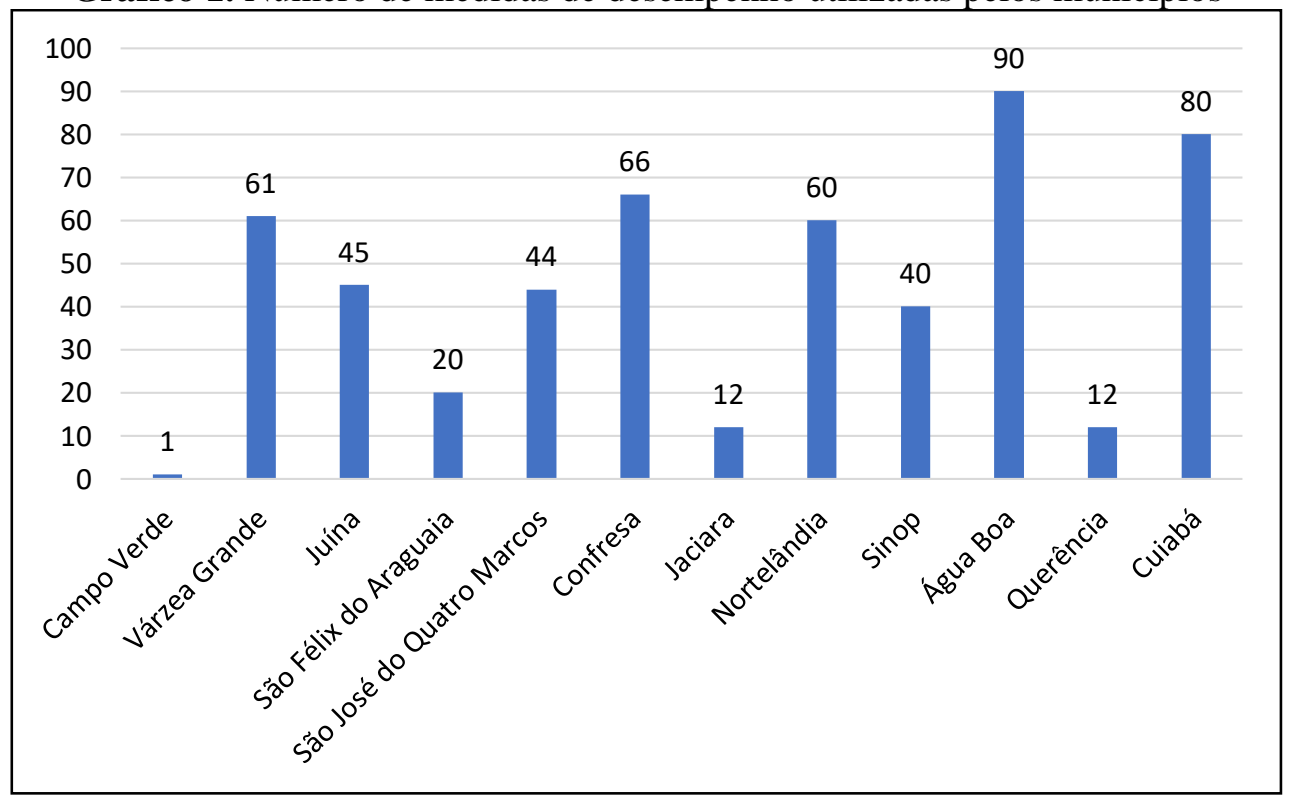

Fonte: Dados da pesquisa

Considerando que o foco do setor público está em gerar satisfação ao cidadão, diferente do setor privado em que o foco é o lucro (BEMFICA; CALLADO, 2016), nesse caso há expectativa de que a perspectiva dos clientes seja considerada mais relevante pelos respondentes, no entanto, a maioria dos gestores dos municípios consideram que as medidas de desempenho financeiras são mais importantes que as medidas de desempenho não financeiras.

O monitoramento dos resultados do BSC é tão importante quanto à sua implementação e desenvolvimento. Tendo em vista que esta é uma ferramenta de controle de estratégias, os resultados precisam ser revisados regularmente. As respostas dos gestores mostraram que $50 \%$ dos municípios realizam o monitoramento mensalmente. A realização da avaliação mensal é um fator considerado positivo, pois está de acordo com a indicação de Lucianetti, Battista e Koufteros (2019) de revisão regular da ferramenta BSC. Cabe destacar ainda, que 16,7 \% dos gestores afirmaram monitorar diariamente os indicadores, ao passo que $16,7 \%$ monitoram a cada 3 meses ou 6 meses a evolução dos indicadores. Em contrapartida os mesmo percentual 16,7\% afirma que monitora apenas anualmente, um prazo longe do ideal.

A partir das respostas dos questionários identificou-se que a maioria dos gestores realizam reuniões a cada três meses para a discussão do planejamento (58,3\%), mas também existe uma frequência positiva para reuniões a cada mês $(16,7 \%)$ e a cada semana $(16,7 \%)$. Apesar de não haver uma definição exata da periodicidade em que as reuniões de planejamento devem acontecer, os resultados obtidos evidenciam que há uma preocupação por parte dos gestores em manter uma maior assiduidade em se discutir o tema. 
De acordo com Lucianetti, Battista e Koufteros (2019), um maior tempo de uso da ferramenta pode demonstrar um BSC com um maior nível de maturidade e com maiores chances de um desempenho organizacional positivo, com geração de resultados melhores. Nesse sentido, o cenário é favorável, já que segundo os respondentes $84 \%$ dos municípios utilizam o BSC a 4 anos ou mais.

Um dos resultados mais relevantes da pesquisa foi em relação à participação da alta administração para a eficácia do BSC na organização, o que é considerado crucial na literatura por Lucianetti, Battista e Koufteros (2019) e Kaplan e Norton (1997), essa importância foi confirmada por aproximadamente $92 \%$ dos gestores.

E por fim, para que uma organização obtenha bons resultados o uso de um sistema de informação eficiente é essencial para que todas as perspectivas estejam alinhadas e todos os indicadores financeiros e não financeiros estejam disponíveis para todos os níveis da organização (NASCIMENTO JÚNIOR; SILVA; MOTA, 2014), porém essa integração pode ser complexa, uma vez que os baixos níveis de integração do BSC com o sistema de informações pode prejudicar a eficácia da ferramenta. Assim quanto a percepção dos gestores em relação a complexidade de integração entre o BSC e os sistemas de informação da organização, identificou-se uma inclinação de respostas positivas, o que demonstra a indicação dos gestores sobre a concordância dessa complexidade de integração nas prefeituras dos municípios de MT. Portanto, essa complexidade precisa ser observada com uma atenção diferenciada pelos gestores que almejam bons resultados.

\section{CONSIDERAÇÕES FINAIS}

O desenvolvimento do presente estudo possibilitou descrever como a ferramenta Balanced Scorecard - BSC tem contribuído para o aprimoramento da gestão pública municipal, a partir da perspectiva dos gestores do planejamento, em 12 prefeituras que integram o Programa de Desenvolvimento Institucional Integrado - PDI do Tribunal de Contas do Estado de Mato Grosso em parceria com a Universidade Federal de Mato Grosso e a Fundação Uniselva.

O estudo identificou na literatura que para uma correta implantação da ferramenta BSC é recomendável a utilização das quatro perspectivas que propiciam um ambiente adequado para o alinhamento estratégico organizacional e o outro aspecto diz respeito as medidas, indicadores, que compõem o BSC, que servem para articulação e comunicação das estratégias.

Nesse sentido, pode-se dizer que, na perspectiva dos respondentes, a ferramenta foi implementada com êxito pelos municípios. Há indicativos de que a ferramenta se encontra em um nível avançado de desenvolvimento onde são utilizadas medidas estratégicas financeiras e não financeiras através de relações de causa e efeito, por meio das quatro perspectivas do mapa estratégico, o que favorece o alinhamento organizacional das prefeituras, contribuindo assim para o aprimoramento da gestão pública municipal.

De maneira geral, os gestores têm uma percepção otimista e satisfatória quanto à aplicação da ferramenta. Na opinião dos respondentes, as prefeituras adotam as perspectivas que compreendem o BSC, comunicam suas estratégias com participação de todos os níveis organizacionais e realizam o monitoramento e atualização frequente dos resultados obtidos por meio do BSC. Um ponto a ser destacado está relacionado aos esquemas de incentivos, pois por se tratar de uma organização pública esperava-se que 
não houvessem recompensas financeiras, entretanto na perspectiva dos participantes elas foram citadas. Esse aspecto pode ser objeto de estudos futuros.

Este estudo tem limitações quanto ao tamanho da amostra e o baixo retorno de respostas, tendo em vista que a amostra inicial era de 23 gestores. $\mathrm{O}$ uso do questionário estruturado limita a captação da percepção dos respondentes, apesar das respostas positivas obtidas. Por fim realizar a análise somente do ponto de vista dos gestores enviesa a análise quanto à correta aplicação e resultados do uso da ferramenta do BSC nos municípios.

Sendo assim, estudos futuros poderiam considerar a perspectiva dos funcionários que possuem ligação direta com o uso da ferramenta BSC, assim como a perspectiva de fornecedores e principalmente dos clientes, nesse caso, os munícipes beneficiários dos resultados gerados pelo uso do BSC como ferramenta de gestão organizacional e estratégica.

\section{REFERÊNCIAS}

ANTONIO, R. et al. A percepção holística da administração gerencial no serviço público. Revista de Administração Geral, v. 1, n. 2, p. 138-153, 2016.

ARAUJO, A. O. Contribuição ao estudo de indicadores de desempenho de empreendimentos hoteleiros, sob o enfoque da gestão estratégica, 2001.

BEMFICA, M. F. C.; CALLADO, A. A. C. Balanced Scorecard no Setor Público : Uma Análise dos Mapas Estratégicos dos Ministérios Públicos Estaduais. Simpósio de Controladoria da UFRPE, p. 1-15, 2016.

BONNEFOY, J. C.; ARMIJO, M. Indicadores de desempeño en el sector públicoSantiagoInstituto Latinoamericano y del Caribe de Planificación Económica y Social, , 2005.

CALLADO, A. L. C. et al. Rentabilidade e Indicadores de Desempenho : uma análise do Setor Hoteleiro segundo as perspectivas do Balanced Scorecard. XV Congresso Brasileiro de Custos, 2008.

CERTO, S. C. et al. Administração Estratégica: Planejamento e implantação da estratégia. 2. ed. São Paulo: Pearson Education do Brasil, 2005.

CHIAVEnATO, I. Administração Geral e Pública: Teoria e mais de 500 questões com gabarito. Rio de Janeiro: Editora Campus, 2008.

COSTA, D. B. Diretrizes para concepção, implementação e uso de sistemas de indicadores de desempenho para empresas da construção civil.Porto Alegre, 2003.

FARIA, A. C. DE; FERNANDES, F. C.; GUIMARÃES, V. M. Balanced Scorecard (BSC) na Gestão Pública: Implementação na Prefeitura Municipal de Ipiranga (PR). XV Congresso Brasileiro de Custos, p. 1-15, 2008.

FRANCO, F.V. et al. Remuneração Estratégica na Administração Pública. Caderno de Administração, v. 25, n. 1, p. 1-10, 2017.

FELIX, R.; FELIX, P. DO P.; TIMÓTEO, R. Balanced Scorecard : adequação para a gestão estratégica nas organizações públicas. Revista do Serviço Público, v. 62, n. 1, p. 51-74, 2011. 
GHELMAN, S.; COSTA, S. R. R. DA. Adaptando o BSC para o setor público utilizando os conceitos de efetividade, eficácia e eficiência. XIII Simpósio de Engenharia da Produção, p. 10, 2006.

GIL, A. C. Métodos e técnicas de pesquisa social. 6. ed. São Paulo: Editora Atlas, 2008.

GODOY, A. S. Introdução à pesquisa qualitativa e suas possibilidades: Uma revisão histórica dos principais autores e obras que refletem esta metodologia de pesquisa em Ciências Sociais. Revista de Administração de Empresas, v. 35, n. 2, p. 57-63, 1995.

GULLO, J. Administração: Para quem estuda, ensina e pratica. 1. ed. São Paulo: Editora Bom Dia, 2016.

HAIR JUNIOR, J. F. et al. Fundamentos de métodos de pesquisa em administração. Porto Alegre: Bookman, 2005.

HELAL, D. H.; DIEGUES, G. C. Do patrimonialismo ao paradigma gerencial: paradoxos na administração pública municipal de Caeté-MG. Administração Pública e Gestão Social, v. 1, n. 1, p. 23-45, 2009.

HELOU FILHO, E. A.; OTANI, N. A utilização de indicadores na Administração Pública: A lei $\mathrm{N}^{\mathrm{o}}$. 12.120/2002 do estado de Santa Catarina. Departamento de Ciências da Administração, 2006.

HERRERO FILHO, E. Balanced scorecard e a gestão estratégica: uma abordagem prática. 10. ed. Rio de Janeiro: Elsevier, 2005.

HITT, M. A.; IRELAND, R. D.; HOSKISSON, R. E. Administração Estratégica. 2. ed. São Paulo: Cengage Learning, 2011.

JESUS, E. DE et al. Indicadores de desempenho na administração pública: Um estudo de caso na diretoria de manutenção da UFRN. XXXIV Encontro Nacional de Engenharia de Produção, 2014.

KAPLAN, R. S.; NORTON, D. P. Estratégia em Ação: Balanced Scorecard. Rio de Janeiro: Editora Campus, 1997.

KAPLAN, R. S.; NORTON, D. P. Organização orientada para a estratégia. 12. ed. Rio de Janeiro: Elsevier, 2001.

LANGWINSKI, P. R. A utilização de indicadores e a medição de desempenho na gestão públicaSanta Maria, 2013.

LUCIANETTI, L.; BATTISTA, V.; KOUFTEROS, X. Comprehensive performance measurement systems design and organizational effectiveness. International Journal of Operations \& Production Management, p. IJOPM-07-2017-0412, 2019.

MATO GROSSO, T. DE C. DO E. DE. PDI - Programa de desenvolvimento institucional integrado: TCE-MT promovendo soluções inovadoras: na linha de sua missão orientadora, compartilha com os fiscalizados a experiência adquirida à partir da adoção do planejamento estratégico e de novas tecnolo. Cuiabá: Tribunal de Contas do Estado, 2013.

MINISTÉRIO DO PLANEJAMENTO, O. E G. Indicadores: Orientações básicas aplicadas à gestão públicaBrasília, 2012. 
NASCIMENTO JÚNIOR, O. R. DO; SILVA, A. P. DA; MOTA, K. J. A. O uso do BSC como ferramenta gerencial no Tribunal Regional Eleitoral de Alagoas. Revista Brasileira de Planejamento e Desenvolvimento, v. 3, n. 1, p. 19-36, 2014.

OLIVEIRA, D. D. P. R. DE. Planejamento Estratégico. 28. ed. São Paulo: Editora Atlas, 2010.

PARMENTER, D. Key Performance Indicators (KPI): Developing, Implementing, and Using Winning KPIs. [s.l: s.n.].

PEDRO, J. M. O Balanced Scorecard (BSC) no Sector Público. Revista Informação e Informática, n. 28, p. 23, 2004.

PEREIRA, J. M. Manual de gestão pública contemporânea. São Paulo: Editora Atlas, 2007.

PEREIRA, M. F. Planejamento Estratégico: Teorias, modelos e processos. São Paulo: Editora Atlas, 2010.

PLANEJAMENTO, M. DO. Guia referencial para medição de desempenho e manual para construção de indicadores. In: Secretaria de gestão. Brasília: [s.n.].

PRODANOV, C. C.; FREITAS, E. C. DE. Metodologia do trabalho científico: métodos e técnicas da pesquisa e do trabalho acadêmico. 2. ed. Rio Grande do Sul: [s.n.].

REBELO, L. M. B.; ALMEIDA, I. G. DE. Planejamento Estratégico : Uma ferramenta de gestão para a administração do setor público estadual do Amazonas. IV Congresso Nacional de Excelência em Gestão, 2008.

RIBAS, A. J. F.; FACINI, M. A.; TEIXEIRA, G. Planejamento Estratégico. Paraná: Gráfica Unicentro, 2014.

RUA, M. DAS G. Desmistificando o problema: uma rápida introdução ao estudo dos indicadores. Avaliação de Resultados no Ciclo da Gestão Pública, p. 1-20, 2004.

SERTEK, P.; GUINDANI, R. A.; MARTINS, T. S. Administração e Planejamento Estratégico. Curitiba: Editora Ibpex, 2007.

SILVA, R. F. P. B. DA. O Balanced Scorecard Aplicado à Administração Pública: Um modelo aplicável a uma instituição de ensino superiorPortugal, 2009. Disponível em: <http://repositorio-aberto.up.pt/bitstream/10216/20596/2/TESEFINAL.pdf>

SILVA, R. R. DA. Importância do setor de recursos humanos no contexto de estratégia da organizaçãoSanta Catarina, 2013.

SILVA, W. C. DA et al. O planejamento estratégico na administração pública: um estudo multicaso. Revista de Ciências Humanas, v. 13, n. 1, p. 90-101, 2013.

SILVA, F. DE A. E; MÁRIO, P. DO C. O processo de formulação e monitoramento do planejamento estratégico de Tribunais de Contas sob a ótica da Nova Sociologia Institucional. Revista de Administração Pública, v. 49, n. 6, p. 1401-1427, 2015.

SILVA JÚNIOR, S. D. DA; COSTA, F. J. Mensuração e escalas de verificação: Uma análise comparativa das escalas de Likert e Phrase Completion. Revista Brasileira de Pesquisa de Marketing, Opinião e Mídia, v. 15, p. 1-16, 2014.

SOUZA, D. L. DE. Planejamento estratégico em organizações pblicas: 
Planejamento de longo prazo em organizações públicas com a utilização do Balanced Scorecard e de cenários prospectivosBrasília, 2010. Disponível em: 〈http://portal2.tcu.gov.br/portal/pls/portal/docs/2507576.PDF>

TEZZA, R.; BORNIA, A. C.; VEY, I. H. Sistemas de medição de desempenho: uma revisão e classificação da literatura. Gestão \& Produção, v. 17, n. 1, p. 75-93, 2010.

TRIBUNAL DE CONTAS DE MATO GROSSO. Competências do Tribunal. Disponível em: <http://www.tce.mt.gov.br/conteudo/sid/876>. Acesso em: 24 set. 2018.

TRIBUNAL DE CONTAS DE MATO GROSSO. TCE lança sistema para facilitar transparência e fiscalização de planos estratégicos de Prefeituras. Disponível em: $<$ https://www.tce.mt.gov.br/conteudo/show/sid/73/cid/46233/t/TCE+lan\%E7a+sistema+ para+facilitar+transpar\%EAncia+e+fiscaliza\%E7\%E3o+de+planos+estrat\%E9gicos+de +Prefeituras>. Acesso em: 5 abr. 2019.

ZANIN, A. et al. Definição de painel de indicadores de desempenho para instituições comunitárias de ensino superior. Revista Gestão Universitária na América Latina GUAL, v. 8, n. 2, p. 2-27, 2015. 